\title{
Concept of solubility, soft drinks and pectin jelly
}

\author{
Arja Ollila \\ Pitkäjärven koulu, Kangasala, Finland • arja.ollila@kangasala.fi \\ Tanja Aho \\ Lahden yhteiskoulu, Lahti, Finland \\ Tiina Helminen \\ Kalevan lukio, Tampere, Finland \\ Anne Helppolainen \\ Lahden yhteiskoulu, Lahti, Finland \\ Katri Mäkelä \\ Pitkäjärven koulu, Kangasala, Finland •ktmakela@kotiposti.net \\ Ville-Pekka Pulkkinen \\ Kalevan lukio, Tampere, Finland
}

\begin{abstract}
The aim of this double class is to demonstrate and emphasise the concept of solubility and associate it with everyday life by preparing and eating simple food in school. This lesson is suitable for upper secondary school and with certain limitations to secondary school. Solubility is discussed in 7th grade chemistry in connection to the study of chemical work methods. In secondary school the work can be used in connection with organic chemistry when learning about sugars and carbohydrates. In upper secondary school concepts detailing substance structure and chemical bonds are further elaborated on and applied to various circumstances.
\end{abstract}

\section{The aim of the workshop}

The aim of the solubility workshop is to enliven the teaching of solubility in secondary school and upper secondary school and to link the concept with the students' everyday life. The workshop is meant to be utilised in upper secondary school and with some adjustments in secondary school. The workshop does not cover all the aspects of solubility so there is room for a deeper handling of the matter.

Solubility of a substance is divided in three parts in this presentation:

- Dissolution of solid substance in liquid

- Dissolution of liquid in liquid

- Dissolution of gas in liquid.

Dissolution of liquid in liquid is discussed only briefly, instead the emphasis is in the dissolution of solid substance and gas in liquid.

One of the aims of chemistry classes in basic teaching is to make chemistry an interesting and experimenting subject. The goal is for the students to learn about and utilise chemical concepts that represent the features of substances, such as solubility.

The aim of the first chemistry course (KE 1) in upper secondary school is for the students to 
learn about different solutions and concepts related to them. One of the principal aims in upper secondary school chemistry is to offer the students experiences that raise and deepen their interest towards the study of chemistry. This workshop provides experiences of chemistry in the context of molecular gastronomy.

\section{Description of the activity}

\subsection{Opening demonstration: What happens to a sugar lump when the sugar dissolves?}

In the start of the workshop a motivational demonstration can be given that helps to initiate discussion. In the demonstration, a sugar lump (sucrose $\mathrm{C}_{12} \mathrm{H}_{22} \mathrm{O}_{11}$ ) is dropped to a glass of lukewarm water. The glass can be placed under a document camera and the image magnified. It is then possible to observe the dissolution for a while and to discuss what dissolution is. Coloured salt, such as potassium permanganate (purple), copper sulphate (blue) or potassium chromate (orange), can be used instead of sugar as a soluble substance. After the demonstration the following matters relating to solubility and dissolution can be discussed:

- Water dissolves substances based on their chemical features.

- As a substance dissolves it spreads evenly to the whole solution. The entire solution has the same amount of the solute's structural units. A solution consists of a solvent and a solute.

- Other factors apart from the features of the solvent and solute also affect the solubility of substances. Generally, as the temperature rises the solubility of a solid substance in water increases and the solubility of gas in water decreases (Figure 1). Small grain size of the solute and stirring the substances accelerates the dissolution process as the interaction between substances enhances.

- Chemical compounds dissolve in structurally similar solvents.

- Solubility relays the amount of solute in a particular saturated solution at a particular temperature. The unit of solubility is generally $\mathrm{g} / 1 \mathrm{or} \mathrm{g} / 100 \mathrm{~g}$.

- There are substances, such as precious metals, that dissolve in hardly any solvent and substances (solid, liquid or gaseous) that dissolve in only certain solvents.

- A solution is considered saturated when at a specific temperature no more of the same substance dissolves in it. 


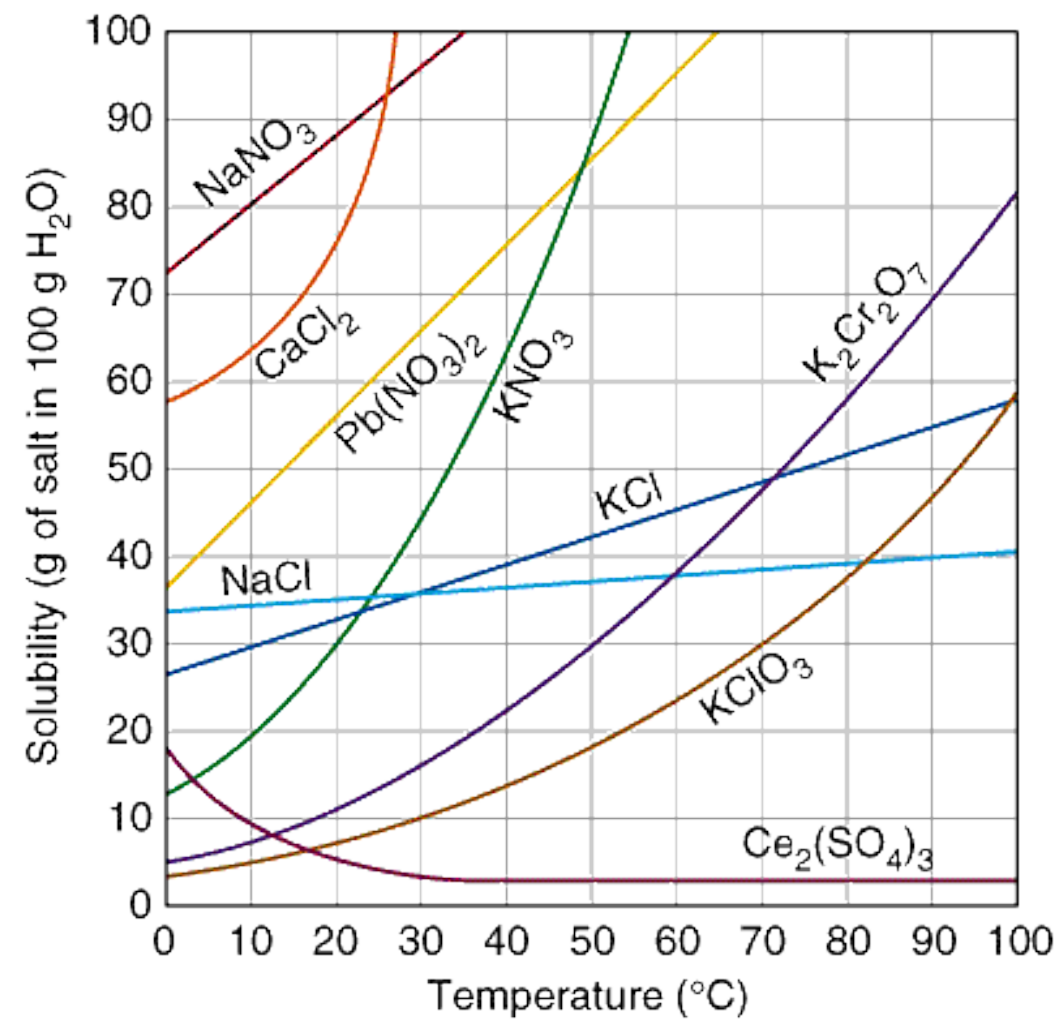

Figure 1. Solubility of certain salts in water at different temperatures. (Source: http://www.sciencegeek.net)

\subsection{Inquiry-based work 1: Cloudberry or raspberry drink}

This inquiry-based work is suitable for 7th - 9th grade chemistry or home economics. The work demonstrates how sugar (sucrose) dissolves in water and how this can be utilised in cooking.

\section{Cloudberry or raspberry drink (4 portions)}

Utensils:

- a $1 \mathrm{dl}$ measuring cup, a kitchen scale, a pot, a wooden fork, a sieve, 4 wine glasses

Ingredients:

- $1 \frac{1}{2}$ dl table sugar

- 300 g cloudberries or raspberries

- 3 dl water

For serving:

- 4- $5 \mathrm{dl}$ sparkling water

- ice cubes 


\section{Method:}

1. Measure the sugar, cloudberries or raspberries and water in a pot. Bring to a boil. Let it simmer at a low temperature for approximately 10 minutes with the lid on. Put the pot in cold water, chill the juice and sift.

2. Pour $1 / 2$ dl of juice in each wine glass. Add cold sparkling water right before serving.

3. You can finish the drinks with ice cubes (Figure 2).

\subsection{Inquiry-based work 2: Raspberry- pomegranate jelly}

This work is suitable for upper secondary school as an example of the solubility of sugar in water and how it can be applied to cooking. The work can be started in the beginning of the lesson as congelation takes time.

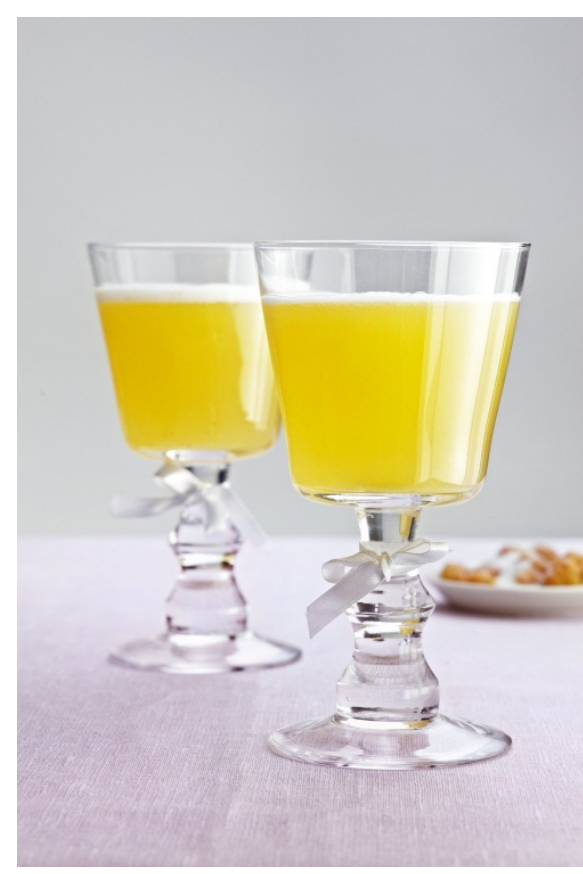

Figure 2. Cloudberry drink. (Reproduced with permission of Suomen Sokeri.)

\section{Raspberry-pomegranate jelly (3- 4 portions)}

Utensils:

- 4 dessert bowls, a $1 \mathrm{dl}$ measuring cup, a vegetable knife, a teaspoon, a pot, a wooden fork

Ingredients:

- $100 \mathrm{~g}$ raspberries (approximately $1 \frac{1}{2} 2 \mathrm{dl}$ )

- 1 pomegranate

- $2 \frac{1}{2}-3 \mathrm{dl}$ water or mild raspberry juice

- $3 / 4 \mathrm{dl}$ gelling sugar

Note: Foreign berries must be heated before use.

\section{Method:}

1. Share the raspberries in dessert bowls. Split the pomegranate and gather the seeds with a spoon and put them with the raspberries.

2. Bring the water to a boil and add the gelling sugar while stirring constantly. Let it simmer until the stock is clear.

3. Pour the stock on top of the berries and put the bowls in the refrigerator. Serve when jelled (Figure 3).

Both fresh and frozen berries can be used in this simple and sophisticated dessert jelly. The berried jelly congeals in the refrigerator in an instance, which makes it perfect for surprise guests. 


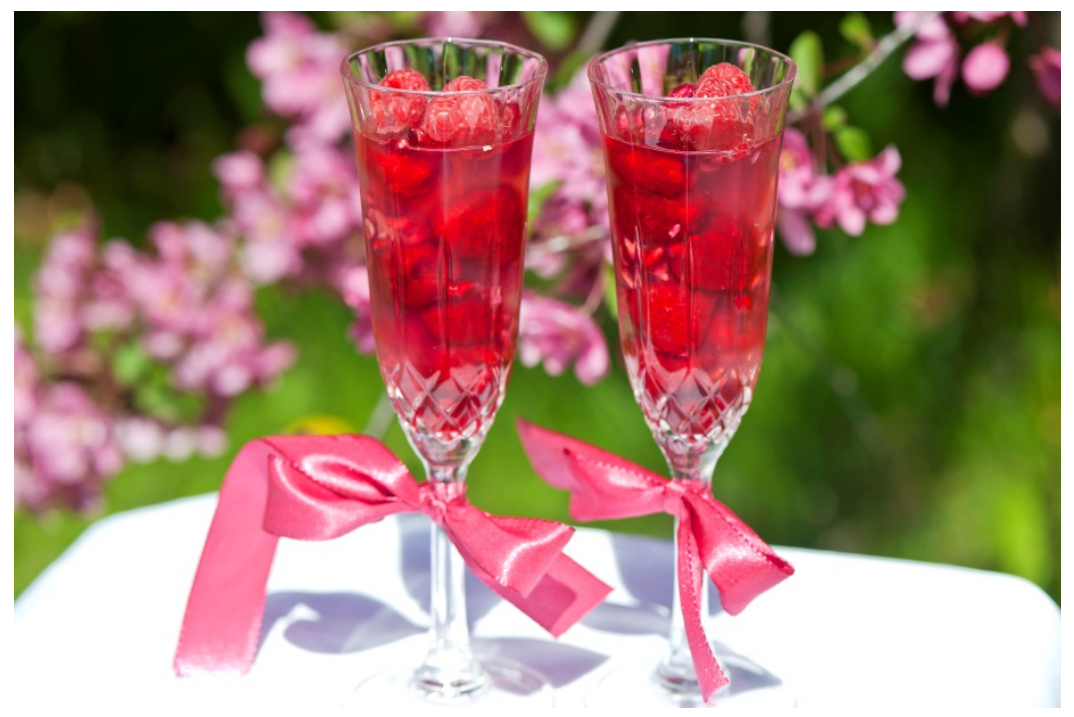

Figure 3. Raspberry-pomegranate jelly. (Reproduced with permission of Suomen Sokeri)

\subsection{Inquiry-based work: Self-made soft drink}

Students tend to enjoy making soft drinks from different juices and fruits, so it is best to reserve time for the activity. The activity requires an appliance that forces carbon dioxide $\left(\mathrm{CO}_{2}\right)$ into water to make it fizzy (SodaStream or similar).

The first step is to carbonate both cold tap water and room temperature (boiled) water and to see if they taste different.

The next step is to try answering the question: why should cold water be used?

The final step is to make a soft drink. J uice squeezed from fruits (orange, lemon, lime, kiwi), ready-made berry juices or juice concentrates (strawberry, cranberry, lingonberry, raspberry) or fruit bits and whole berries, even cucumber, can all be used for the drinks. Sugar can also be added if so preferred.

\subsubsection{What is the use or joy in making one's own soft drink?}

While tasting the drinks it is possible to discuss self-made drinks in the context of sustainable development. It is good to know the origin of the ingredients and personally to be able to adjust the sugar content. The amount of plastic waste decreases as well. Energy and time is saved by not having to go and buy the drinks from a shop. It is worthwhile to discuss the environmental impact of home carbonation products and carbon dioxide cylinders. With the proper use of the appliance and by recycling the carbon dioxide cylinders the environmental impact during use can be lowered. 


\section{The chemical background}

\subsection{Dissolution of solid substance in liquid}

\subsubsection{Dissolution of sucrose, or table sugar, in water}

The water molecule is a polar molecule that has partial charges; oxygen has a negative charge $\left(\delta^{-}\right)$and hydrogens have a positive charge $\left(\delta^{+}\right)$. Because of this, water molecules attract with weak electric charges other water molecules (Figure4) and other charged molecules. These attractive forces are called hydrogen bonds or hydrogen bridges.

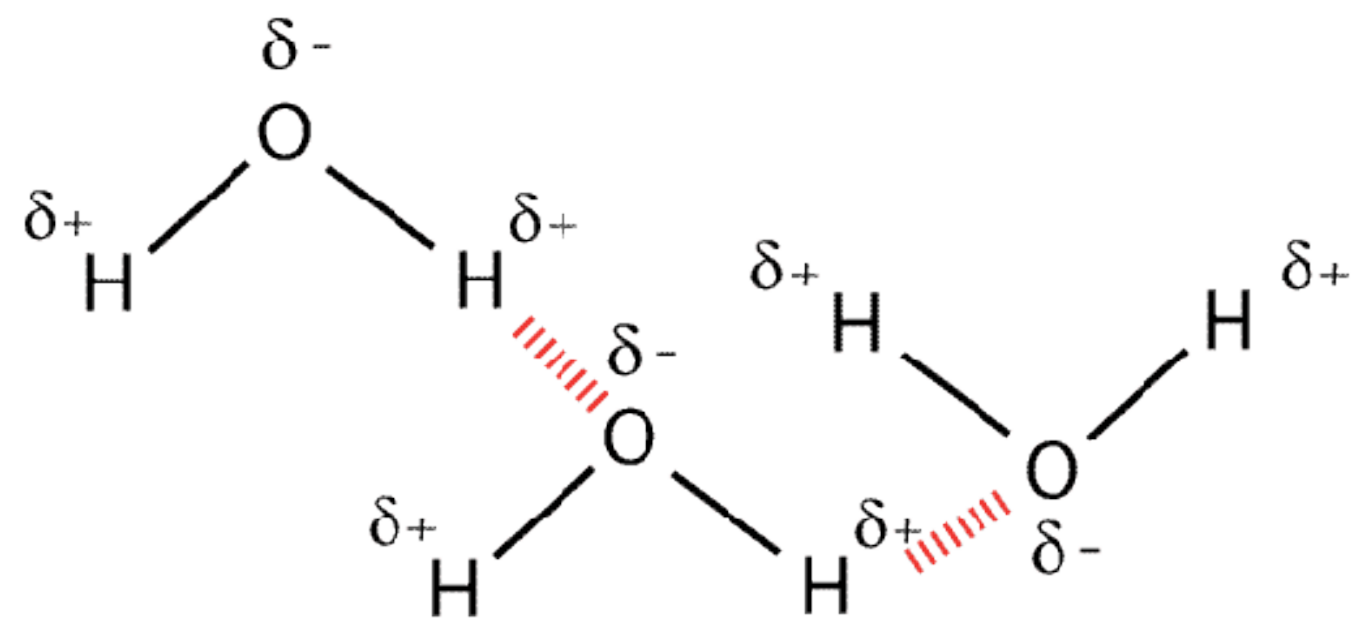

Figure 4. Hydrogen bonds or hydrogen bridges between water molecules.

(Source: http://chemtools.chem.soton.ac.uk/projects/emalaria/index.php?page=13l)

Sugars are small molecule, sweet and easily water soluble carbohydrates. Because of their hydroxyl functional groups $(\mathrm{OH})$ sucrose molecules are electrically segmented similarly to water molecules (Figure 5). Like water molecules, sucrose molecules also form hydrogen bonds with each other.

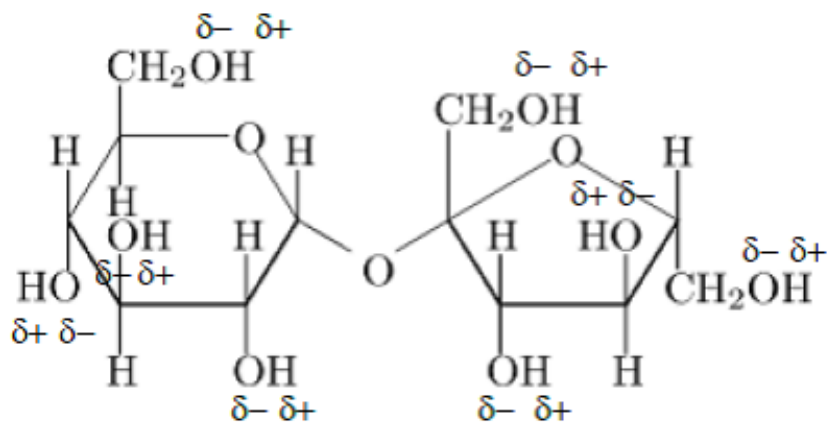

Figure 5. Structural formula and partial charges of the sucrose molecule. (Source: http://upload.wikimedia.org/wikipedia/commons/8/8c/Saccharose.png) 
Water effectively dissolves substances that are in the same way polar as water: "substance dissolves similar substance." Water can separate hydrogen bonded sugar molecules from each other with its attractive forces (Figure 6). The outer molecular layer comes off first. As it disperses, the next layer disengages to the liquid and so forth. Stirring accelerates the dissolution process because it increases the impact speed of the molecules.

The higher the water temperature, the more sugar dissolves in water. As the temperature rises, the kinetic energy of water molecules increases. Water molecules encounter sucrose molecules which they separate from each other faster if the impact speed is sufficient and the angle of impact right. Individual separated sucrose molecules disperse in with the water molecules.

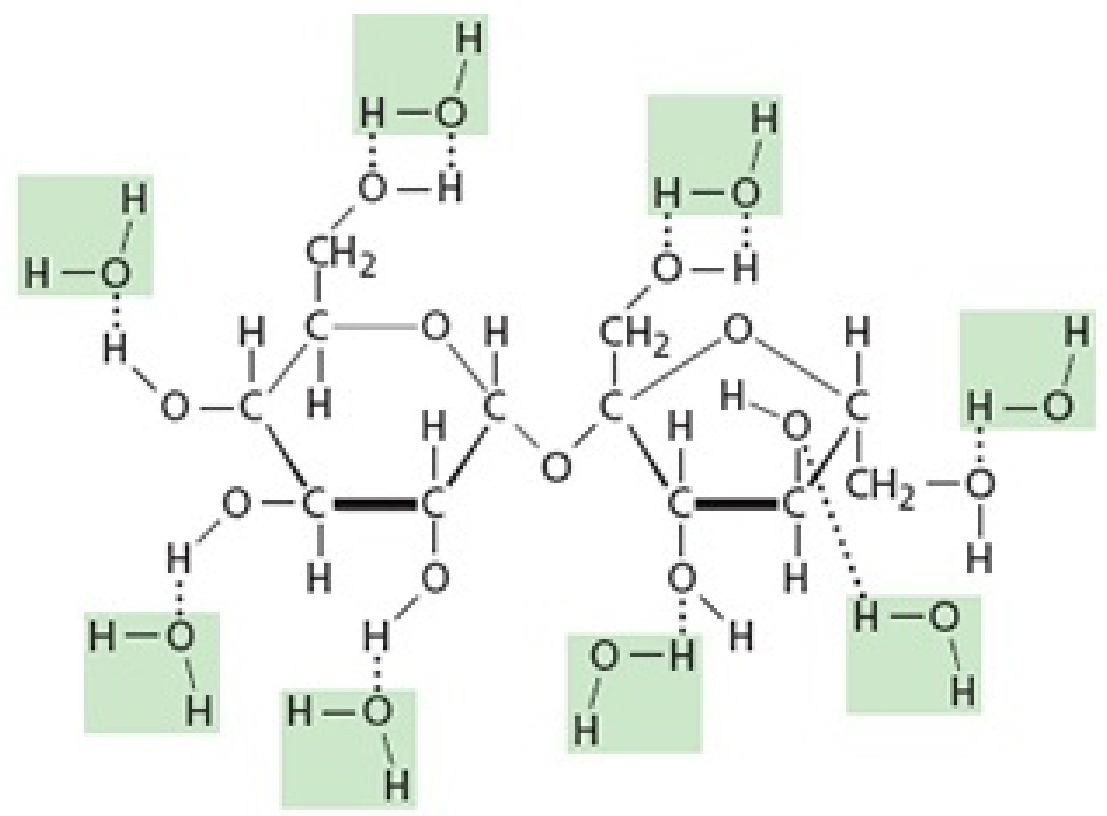

Figure 6. Dissolution of a sucrose molecule in water. (Source: http://glencoe.mcgraw-hill.com/sites/dl/free/007874637x/514787/fig14_12.jpg)

\subsubsection{Dissolution of pectin in water}

Polysaccharides are long-chain carbohydrates. Polysaccharides are insoluble in water but they are able to bind large amounts of water because of their $\mathrm{OH}$ groups. Pectin is a longchain polysaccharide (Figure 7) that is used to form jellies. Gelling sugar contains sugar (sucrose) and pectin as a gelling agent. Pectin requires $90{ }^{\circ} \mathrm{C}$ temperature to dissolve in water. When the solution cools down it forms gelatinous half-solid mass. J elly is formed when the pectin's long carbohydrate chains bind to each other through hydrogen bridges and form a netlike structure. Water is bonded inside the pectin network. 


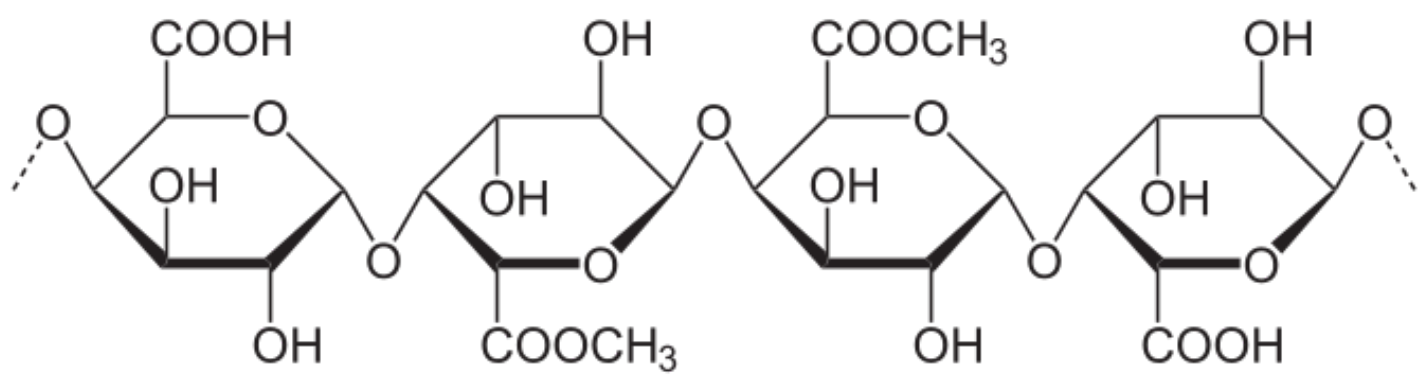

Figure 7. Pectin molecule structure.

(Source: http://commons.wikimedia.org/wiki/File:Pektin2.svg)

\subsection{Dissolution of liquid in liquid}

Polar liquids dissolve in polar solvents and nonpolar liquids in nonpolar solvents. This phenomenon is utilised in making alcoholic drinks, for example. Water molecules and ethanol molecules $\left(\mathrm{C}_{2} \mathrm{H}_{5} \mathrm{OH}\right)$ are structurally similar, they are both polar and dissolve into each other endlessly; the solution never becomes saturated.

\subsection{Dissolution of gas in liquid}

Generally, the solubility of gas in liquid decreases as temperature rises (Figure 8) but increases as pressure increases.

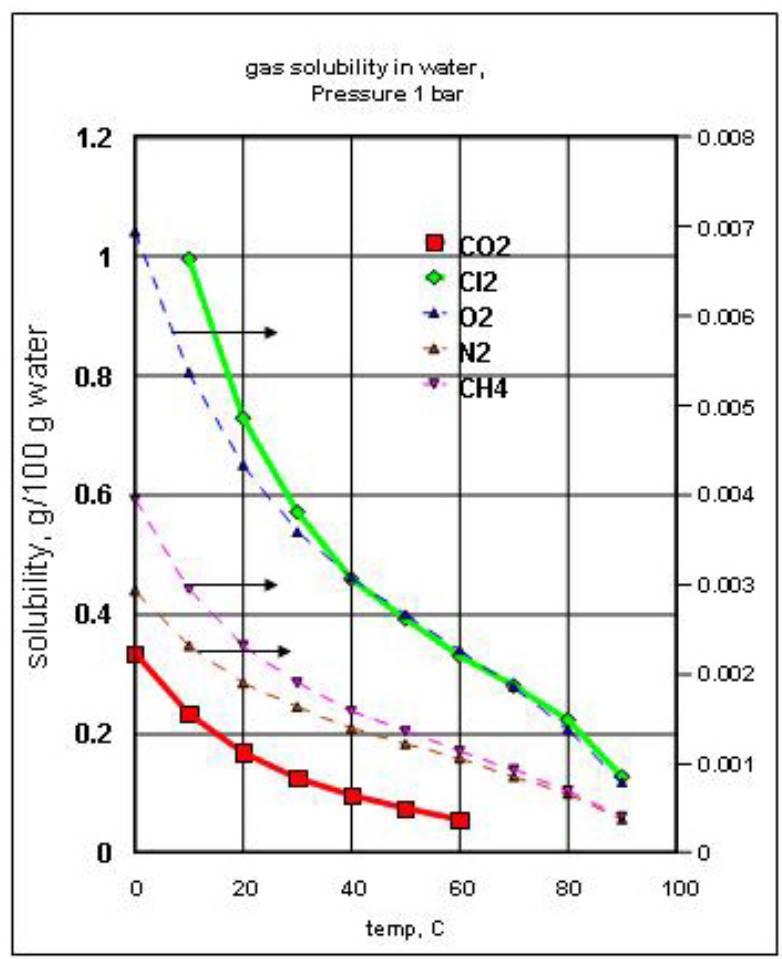

Figure 8. Solubility of certain gases in water at different temperatures.

Source: http://www.oup.com/us/static/companion.websites/9780195159172/chapter6/ch6-fig2.html 


\subsubsection{Dissolution of carbon dioxide in water}

Carbon dioxide gas $\left(\mathrm{CO}_{2}\right)$, used in making the soft drink, dissolves more easily in cold water than in hot water (Figure 8). The gas bubbles stay between water molecules better in cold water than in hot water, where they expand and diverge from the solution faster. 
OLLILA ET AL. 